\title{
Tsafon
}

Revue d'études juives du Nord

$80 \mid 2020$

Varia

\section{Waysand Georges, Profession du père : fusillé}

\section{Monique Heddebaut}

\section{(2) OpenEdition}

\section{Journals}

Édition électronique

URL : https://journals.openedition.org/tsafon/3437

DOI : $10.4000 /$ tsafon.3437

ISSN : 2609-6420

\section{Éditeur}

Association Jean-Marie Delmaire

\section{Édition imprimée}

Date de publication : 1 décembre 2020

Pagination : 169-176

ISSN : 1149-6630

\section{Référence électronique}

Monique Heddebaut, «Waysand Georges, Profession du père : fusillé », Tsafon [En ligne], 80 | 2020, mis en ligne le 01 décembre 2020, consulté le 21 septembre 2021. URL : http://journals.openedition.org/ tsafon/3437 ; DOI : https://doi.org/10.4000/tsafon.3437 


\section{Lecture ......}

Waysand Georges, Profession du père : fusillé, Lormont, Éd. Le Bord de l'eau, 2020, 318 p., $25 €$.

Tranches de vie : par Monique Heddebaut

«Profession du père: fusillé », c'est ainsi que Georges Waysand remplissait l'inévitable fiche de renseignements à chaque rentrée scolaire. De ce père - Mojsze Chaïm Waysand, Maurice, Mouni ou Jean, selon les circonstances - venu de Pologne, il n'en garde pas de souvenir conscient. Le fils est alors parti sur les traces d'un père absent, invisible derrière l'image conventionnelle du héros, pourtant resté omniprésent. Dans un style éminemment personnel, très foisonnant ${ }^{1}$, dans un parcours intime où il dialogue avec lui-même, il mêle, entremêle informations et sentiments dans une recherche historique détaillée sur ses parents et sur leur implication dans le mouvement communiste et la Résistance. Le lecteur l'accompagne dans cette reconstitution et dans ce voyage qui le mène de la Pologne à la Belgique, au Nord de la France et même en Espagne lorsqu'il s'agit de sa mère Esther Zilberberg, dite Estoucha ${ }^{2}$. Georges Waysand est parti d'un album photos, de quelques papiers conservés par celle-ci. Il les a disséqués, analysés, cherchant à les faire parler, en scientifique qu'il est. L'auteur qui est rodé à la pratique exigeante de la recherche est physicien et historien de la supraconductivité. Son investigation se dévoile alors comme une série de poupées gigognes: ce sont plusieurs voyages à l'intérieur d'une même quête, d'un même voyage. Ce fut une descente dans les fonds d'archives de cette période en Belgique et en France, qui furent si longues à s'ouvrir et qui mettent désormais en lumière les mécanismes de surveillance mis en place par les autorités allemandes et françaises, comment eurent lieu les traques, les arrestations, les interrogatoires, les collaborations qui aboutirent aux exécutions. Il a confronté les témoignages, est reparti sur les lieux. Il s'est appuyé sur une solide bibliographie y compris sur les travaux plus récents tant belges que français.

Son père, Jean Waysand, est né en 1916 à Lodz puis, après la guerre, sans doute après le traité de Versailles, s'est installé à Toruń en Poméranie (Pologne), ville encore allemande avant la Première Guerre mondiale. La plupart des juifs de cette ville étaient d'ailleurs de culture allemande. Le père est relieur à son compte et la mère au foyer. Il a une sœur Mania ${ }^{3}$. Jean arrive en Belgique avec un visa établi le 30 septembre 1938 et un

\footnotetext{
${ }^{1}$ Dans la partie «Noms cités» en fin de volume, l'auteur aurait pu mettre en regard noms et numéros de pages, proposant ainsi un véritable index. Une partie rassemblant toutes les cotes des archives consultées aurait également été la bienvenue.

${ }^{2}$ Estoucha, Paris, Denoël, 1997, 438 p.

${ }^{3}$ Cette famille compte sans doute d'autres enfants, selon Georges Waysand.
} 
diplôme de dessinateur industriel en poche, obtenu à Varsovie la même année. Il quitte la famille le 23 octobre, bien décidé à entamer une formation d'ingénieur à Bruxelles, là où vit Herz Adler, le frère de sa mère, depuis 1925. Ce dernier était revenu au pays en 1926 à Lodz épouser Jenta Lajerowicz avec laquelle il a trois enfants ${ }^{4}$. Il est à la tête d'une petite entreprise de couture en gros, manteaux et tailleurs, au 106 rue Vondel à Schaerbeek-Bruxelles, ce qui lui permet de prendre en charge son neveu comme il le déclare le 13 juillet 1938. Jean y reste jusqu'au 17 avril 1940, date à laquelle il part rue du Vautour selon le Bulletin de la Police des Étrangers. Son engagement n'est pas récent puisque, déjà en Pologne, il appartient à des groupes sionistes très à gauche, quasiment marxistes, le Hashomer Hatzaïr, un mouvement né en 1913 en Autriche. Pour ce fils d'artisan issu de la petite bourgeoisie, l'ascension sociale va de pair avec une déjudaïsation progressive.

Sa future compagne, Esther Zilberberg, dite Estoucha, est née en 1912 à Kalisz, une ville à l'ouest de Lodz, dans une famille de cinq enfants. Le père est ouvrier dentellier'5 Elle décide en 1929 de partir en Belgique pour y entreprendre des études de médecine. N'ayant pas assez d'argent pour aller plus loin, elle arrive à Liège et s'inscrit à la faculté de médecine tout en travaillant pour gagner sa vie. Dans un de ses petits boulots, elle participe au déclenchement d'une grève, est licenciée et n'arrive plus à trouver de travail. Son amie d'enfance, Lola, qui est déjà à Bruxelles, l'aide à la rejoindre. Elle s'inscrit alors à l'ULB (Université Libre de Bruxelles). Ce choix est au diapason des orientations politiques et éthiques de cette université. Fondée en 1834 par un avocat, franc-maçon notoire, Pierre-Théodore Verhaegen (1796-1862), et créée en réaction contre le monde clérical, elle a pour objectif de répandre la philosophie des Lumières ${ }^{6}$. En 1938 le Cercle de Médecine avait affiché sur son char dans la plus pure tradition carnavalesque belge ses positions antifascistes avec des caricatures des rexistes et de nazis.

L'un et l'autre ont quitté la Pologne où la vie devenait de plus en plus difficile, en raison de la profonde misère, de l'antisémitisme croissant et des quotas imposés dans les écoles et les universités. Jean a choisi le chemin de l'exil quelques jours avant la « Nuit de cristal ». Il n'y eut aucun survivant dans leurs familles respectives restées en Pologne. Ils arrivent en Belgique, dans un pays où le nombre de juifs est estimé à 70000 en 1939, et dont la majeure partie - sans doute près de $95 \%$ - n'a pas la nationalité belge ${ }^{7}$. Estoucha croise, à Bruxelles, Jean qui sera indissociable de sa vie, mais le couple s'est formé plus tard, sur les chemins de l'exode. Elle est membre du Parti Communiste Belge (PCB) depuis 1934, d'abord par antifascisme. Ce parti compte « à peu près dix mille membres pour une population de 7,7 millions [...]. Vingt pour cent du PCB étaient juifs alors que la Belgique ne comptait que 65000 juifs ${ }^{8}$.

Éclate alors la guerre civile en Espagne en juillet 1936. Estoucha part dès les premiers jours, le 8 août 1936, avec un doctorant en biologie, Abraham Gostynski, pour soutenir les républicains espagnols. Celui-ci est tué trois jours après leur arrivée à Irun. Elle participe au service sanitaire du bataillon basque Perezagua sous le nom de JuanitaJeanne Lefebvre, dans les premières lignes du front basque. Légèrement blessée au

\footnotetext{
${ }^{4}$ Jenta Lajerowicz disparaît à Auschwitz avec ses trois enfants.

${ }^{5}$ Dans un autre texte écrit par Estoucha, on comprend qu'il est plutôt comptable ou employé aux écritures.

${ }^{6} \mathrm{La}$ St-V ou Saint-Verhaegen reste fêtée chaque 20 novembre par les étudiants de l'ULB.

${ }^{7}$ Rudi Van Doorslaer (dir.) Emmanuel Debruyne, Frank Seberechts, Nico Wouters Avec la collaboration de Lieven Saerens, La Belgique docile Les autorités belges et la persécution des Juifs en Belgique pendant la Seconde Guerre mondiale https://www.senate.be/event/20070213jews/doc/rapport_final.pdf, p. 46.

${ }^{8}$ Estoucha, Paris, Denoël, 1997, p. 46. La note 1 cite Rudi Van Doorslaer, dans La Belgique docile..., op. cit., p. 60.
} 
combat au printemps 1937, elle ne retourne pas au front. Ayant adhéré au PC espagnol elle devient journaliste au journal républicain pour femmes Mujeres. Elle participe ensuite à l'évacuation des soldats et des civils, en tant que médecin, vers Bordeaux. Revenue en Espagne côté catalan, elle devient interprète russo-espagnole à bord d'avions de chasse soviétiques, car l'URSS s'est engagée par l'intermédiaire du Komintern en livrant des armes aux républicains. Elle franchit la frontière espagnole le 9 février 1939 et Madrid tombe le 28 mars : la guerre civile prend fin. S'organise alors le retour des illégaux vers leurs pays de départ. Radiée d'office par la Pologne qui a retiré leur nationalité à tous ceux qui ont combattu en Espagne, elle conservera son autorisation de séjour définitif en Belgique.

De passage à Paris, elle fait appel à ceux qui ont combattu en Espagne et aux solidarités communistes et juives. Elle est brièvement hébergée chez Régine et Arek Kowalski, des communistes polonais. Arek est un ancien brigadiste. Elle s'installe ensuite chez l'un des cousins de sa mère, Maurice Selver au 37 rue Censier ( $5^{\mathrm{e}}$ arrt). Elle se tourne vers le Comité d'aide aux anciens d'Espagne dont le secrétariat est assuré par deux communistes, André Heussler et Jules Dumont ${ }^{9}$. Les contacts passent aussi par l'ULB, la faculté de droit et des Bruxellois, deux socialistes d'avant-garde, l'avocat Marc Somerhausen, député de Verviers, et Henri Rolin, sénateur, professeur de droit international à l'ULB. Elle dépose à Paris une demande de séjour définitif en France, car elle aurait « perdu ses papiers ». Elle utilise plusieurs adresses dans ses démarches : le 113 rue du fg Poissonnière, le siège du Comité d'Aide aux Volontaires Anciens combattants, blessés, mutilés, prisonniers et orphelins de l'Espagne républicaine, celle de Sarah et David Dwelajki ${ }^{10}$, et le 78 rue de Pixérécourt. Cette adresse correspond à un lieu de réunions d'organisations juives, au siège du Yddisher Arbeiter Sport Klub (YASK) [Club sportif ouvrier juif] et au bureau de recrutement des Brigades internationales. Elle renoue des liens avec ses amies juives arrivées à Paris : Lola Optowski et son mari Isaac, Zosha Poznanska ${ }^{11}$ et Schmulek, son compagnon parisien...

Restée à Paris jusqu'au 3 août 1939, elle revient finalement à Bruxelles, à l'ULB, qui peut lui offrir une bourse d'études. Court répit jusqu'à l'invasion allemande de la Belgique et de la France le 10 mai 1940. Jourdain, son directeur de labo, lui conseille « avec le nom qu'elle porte [...] de foutre le camp». C'est sur les lieux de l'exode, à Montoulieu-Saint-Bernard (Ariège), non loin de Toulouse, après juin 1940, que Jean et Estoucha deviennent intimes, un «défi au milieu du désastre ». L'un et l'autre avaient pris l'un des derniers trains quittant Bruxelles... Le fils de l'ancien maire de la commune a pu nommer à l'auteur presque tous les membres d'une photo de groupe, de ces réfugiés juifs polonais de Belgique, scouts catholiques français venus en vélo de Paris, dames belges et leurs enfants... La région hébergeait déjà des dizaines de milliers de réfugiés et d'exilés. Elle qui était interne en médecine, remplace le médecin mobilisé dans une commune proche, Saint-Martory. Toulouse, terminus des trains d'évacués, devient un lieu privilégié pour les réseaux en cours de formation.

C'est ensuite le retour en Belgique, de là où ils étaient partis, obéissant ainsi aux consignes des appareils de direction des partis communistes belges et français. Mais le couple transite par Paris car Estoucha doit impérativement remettre, sans plus de précisions, des documents confidentiels à un copain étudiant de Bruxelles. S'agit-il de listes d'internés, de leurs biographies, de rapports d'activités ? Le 17 novembre 1940, le $23^{\mathrm{e}}$ anniversaire de la Révolution d'Octobre est fêté dans l'intimité d'un atelier de

\footnotetext{
${ }^{9}$ Jules Dumont a été fusillé au Mont-Valérien le 15 juin 1943.

${ }^{10}$ David et Sarah Dwelajki ont été déportés par le XIX ${ }^{\mathrm{e}}$ convoi parti de Malines le 15 janvier 1943.

${ }^{11}$ Zosha Poznanska s'est suicidée à la prison de Saint-Gilles en 1942.
} 
maroquinerie des parents d'une camarade selon les consignes du PCB alors que le pacte germano-soviétique est toujours en vigueur, ce qui a créé un profond malaise chez les communistes belges et français et des démissions. Les directives officielles du Kominterm - IIIe Internationale - ne sont-elles pas encore à cette date de lutter contre les impérialistes franco-anglais et allemands? Le couple s'installe au 131 avenue du Roi à Forest, dans la banlieue sud-ouest de Bruxelles. Lui est étudiant ingénieur à l'Institut National des Industries de Fermentations Industrielles, un vivier de sommités scientifiques et de prix Nobel. Il se présente le 22 janvier 1941 à l'examen de passage en $3^{\mathrm{e}}$ année en vue du grade d'ingénieur-technique, spécialité meunerie. Tous deux fréquentent les milieux des réfugiés politiques, des immigrés bruxellois et des membres du cercle des étudiants marxistes de l'ULB où ils s'étaient rencontrés.

Ils ont la volonté d'officialiser leur situation en publiant les bans le 9 mars 1941, mais le mariage n'aura jamais lieu en raison d'une actualité de plus en plus tendue et des manifestations qui s'enchaînent. Une semaine plus tard le PCB et la Jeune Garde Socialiste manifestent contre la tenue au Cirque royal d'un meeting du Vlaams National Verbond, la Ligue Nationale flamande, qui collabore avec l'occupant. Un meeting communiste est organisé au Vieux Marché pour chasser les fascistes des quartiers ouvriers et des boulevards centraux. Mais la rupture du pacte germano-soviétique donne un coup d'accélérateur à la guerre, également à la traque de tous les opposants. L'opération Sonnenwende est déclenchée et des centaines de communistes, socialistes de gauche, trotskystes sont arrêtés après le 22 juin 1941. Le parti communiste français entend développer l'action directe et organiser la lutte armée pour une partie des militants dès l'été 1941. La décision de l'organisation clandestine de la Main-d'Oeuvre Immigrée (MOI) en Belgique est tout aussi claire. Est alors formée la compagnie juive du Corps mobile des Partisans armés qui abat le 29 août 1942 Robert Holzinger, indicateur juif au service de l'occupant, tandis que l'Association des Juifs en Belgique $(\mathrm{ABJ})$ se trouve impliquée dans la mise en œuvre des déportations vers Auschwitz. Les communistes belges et sionistes de gauche ont dénoncé son rôle. Le consensus de la politique du «moindre mal » dans lequel s'était installée la Belgique occupée après mai 1940, est rompu. Le couple déménage clandestinement à Anderlecht, au 30 rue de l'Émulation. L'ULB qui subit des pressions externes et internes, préfère se saborder en novembre 1941 plutôt que de se mettre au pas et de se soumettre aux professeurs d'Ordre nouveau.

Leurs itinéraires vont ensuite se déplacer vers le Nord de la France qui appartient au MilitärBefehlshaber für Belgium und Nordfrankreich [Commandement militaire pour la Belgique et le Nord de la France], le MBB, depuis juin 1940. Jean est arrivé en avril 1941 dans le Cambrésis, avant la naissance de son fils. Il est embauché par l'entreprise Maye, en tant qu'électricien, magasinier. En prévision d'un débarquement sur les côtes du Pas-de-Calais, les autorités d'occupation qui développent un aérodrome à Niergnies à $5 \mathrm{~km}$ au sud de Cambrai, ont grand besoin de main d'œuvre. 15000 ouvriers y ont travaillé. Tout un réseau de résistants se crée autour des bases aériennes, avec les frères Beauvois à Épinoy et Guillaume Ferrari, son camarade de travail à Niergnies. Le premier acte résistant de son cousin Eusebio Ferrari fut de faire flotter au sommet d'un pylône un drapeau rouge avec un marteau et faucille à Fenain, le matin du $1^{\text {er }}$ juillet 1940. Guillaume a combattu dans les Brigades internationales avec quatre autres Italiens du Valenciennois. Jean devient responsable technique Nord/Pas-de-Calais de la résistance communiste. Les missions sont diverses, mais surtout techniques : sortir du matériel de la base de Niergnies, transporter des explosifs, étudier des dispositifs de mise à feu, faire des essais dans la courette du logement, afin de découvrir les façons les plus efficaces dans les sabotages des voies ferrées, des écluses. Les cheminots moyennement sûrs de l'efficacité des explosifs, préféraient déboulonner une éclisse et des tirefonds plutôt qu'une charge sous le rail. Les résistants sont également charger de 
récupérer des tickets d'alimentation dans les mairies. À Thun-l'Evêque un affrontement a causé la mort de l'un des employés de mairie.

Le couple a emménagé à Cambrai, 4 rue Saint-Géry, vers le $1^{\text {er }}$ août 1941 , adresse découverte en octobre 1942 par la gendarmerie de cette ville. Témoignage éminemment émouvant : Guillaume Ferrari a raconté à Georges Waysand l'avoir tenu dans ses bras. Estoucha entreprend des démarches sous le nom de Zilberberg pour obtenir une carte de ravitaillement. Sur un document daté du 12 août 1941 le nom Waysand, devenu terrassier entretemps, est ajouté. Ils partent rue Bertrand Milcent. Les Dwelajki venus fin août 1941, juste avant le couvre-feu imposé aux juifs en Belgique, s'empressent de repartir pour ne pas tomber sous le coup de la loi anti-juive.

L'étau se resserre autour de Jean et du couple. En raison de la recrudescence des sabotages dans le Douaisis et le Valenciennois, le préfet Carles décide début janvier 1942 de détacher plus de 300 gendarmes pour organiser des patrouilles et surveiller les installations industrielles. Il n'a pas détruit les fichiers des Renseignements généraux à la différence de certains de ses collègues, ce qui va faciliter la tâche des Allemands dans leur poursuite des résistants. En juin 1942 Jean, doit «passer dans le brouillard», formule qui exprime le passage dans la clandestinité. Il loge désormais dans la cité minière de Dessevalle à Somain (Nord) chez Alexandre Bisiaux ${ }^{12}, 46$ allée D depuis 15 jours, début septembre. Il y a entreposé du matériel. Le domicile est perquisitionné, une souricière est tendue. Le 19 septembre 1942, une semaine après la rafle du 11 septembre des juifs du Nord/Pas-de-Calais, il est arrêté à Somain par la gendarmerie française. En Belgique, le couple s'était enregistré au fichier juif, ce qu'il n'avait pas fait en France. Jean est emprisonné et condamné à mort par le tribunal allemand FK 678 de Valenciennes. Il est transféré le 24 à Loos, après un bref séjour de quelques jours à Cuincy, près de Douai. L'enquête révèle qu'il a une concubine espagnole, Juanita Alvarez, et un enfant. Le seul compagnon de cellule qui ait survécu, un médecin résistant, Denis Cordonnier ${ }^{13}$, a raconté à Estoucha les dernières heures de Jean, fusillé le 15 décembre 1942 au fort du Vert-Galant à Wambrechies (Nord) avec cinq autres résistants. Il appartient au martyrologe communiste où sont inscrits quatre-vingtquatorze noms parmi lesquels trente-cinq sont les otages fusillés dans ce fort après la grève des mineurs de mai-juin 1941. Plus de la moitié des douze résistants fusillés du 15 décembre 1942 avaient été en contact avec lui.

L'auteur, Georges Waysand, le "gosse », l'enfant caché, est né le 30 avril 1941 à l'hôpital Saint-Pierre rattaché à la faculté de médecine de l'ULB. Le père n'a pu se rendre à Bruxelles que le 4 mai, car il travaillait dans le Cambrésis. Jeanne et Fernand lui ont tout dit de son enfance, c'est-à-dire le peu dont ils se rappelaient sur son père. Fernand était souffleur de verre. L'enfant est placé chez une femme de Boussois dont le mari était prisonnier et reçoit des Allemands. Il y est mal soigné, à la limite de la maltraitance. Ses parents prennent la décision en juin 1942 de reprendre l'enfant alors âgé de 14 mois. Mais après cet essai infructueux et trop risqué de six semaines, les parents n'ont plus nulle part où le placer. Début juillet 1942, ils se tournent vers un couple de camarades sûrs, Jeanne et Fernand Deroubaix, qui habitaient, avec leur fille Renée (orpheline du premier mari de sa mère) âgée de huit ans à La Bassée, près de Lille. Après l'armistice Martha Desrumaux qui assurait la liaison entre Bruxelles et les communistes du Nord, passait de temps à autre chez Jeanne et Fernand où elle dormait. Sur cette terre où se battirent troupes anglaises et allemandes pour le contrôle de La Bassée pendant la Première Guerre mondiale soufflait l'esprit de résistance. Jean et

\footnotetext{
${ }^{12}$ Alexandre Bisiaux a été fusillé au Mont-Valérien le 18 juin 1943.

${ }^{13}$ Denis Cordonnier, maire socialiste de Lille à la Libération et décédé en 1952, a publié ses souvenirs de la section allemande de la prison de Loos-lez-Lille : Dans les Geôles allemandes (Loos 1942-1943), Avesnes, impr. de l'Observateur, 1945, 154 p.
} 
Estoucha sont arrivés ensemble à La Bassée. Après avoir déposé Georges chez les Deroubaix, les parents n'auront évidemment plus de refuges partagés. Il ne fallait plus « dormir à plusieurs dans une même planque »: les consignes étaient impératives. Les visites de l'un ou l'autre parent chez Jeanne et Fernand furent brèves ensuite. Jean/Maurice n'y repassa qu'une seule fois.

Estoucha, arrêtée le 6 février 1943, est déportée à Ravensbrück et à Mauthausen. Lorsqu'elle retrouve son fils en mai 1945, au début, celui-ci ne comprend pas qui elle est. Elle l'a emmené à l'été 1946 pour des vacances en Suisse et placé ensuite dans des maisons d'enfants, d'abord à Sainte-Maxime, puis à Nice grâce la Commission Centrale de l'Enfance de l'Union des Juifs pour la Résistance et l'Entraide (UJRE) ${ }^{14}$. Elle n'a pu avoir un studio à elle qu'en mai 1948, ce qui explique que son fils n'ait quitté définitivement La Bassée que fin octobre 1948. L'auteur parle d' « une enfance heureuse pendant la guerre » dans ces terres du Nord.

Un autre intérêt de ce livre est de nuancer les rapports de la population avec les Allemands et d'y apporter un éclairage peu étudié, cette fois sous l'angle des consignes des partis communistes envers l'occupant. Des logements avaient été réquisitionnés pour les soldats allemands du génie cantonnés à La Bassée, le temps de reconstruire un pont provisoire dans cette région qui avait souffert des bombardements massifs lors de la bataille d'Haubourdin en mai 1940. Les Deroubaix sont obligés de loger trois soldats. Les rapports sont corrects, sans animosité particulière. «On voyait bien que c'était des ouvriers comme nous. On avait reçu des consignes d'essayer de sympathiser avec eux et même d'organiser des matchs de football » selon Fernand. Ce sont des fermiers en fait. L'un d'eux, August, partage à l'occasion son colis avec la famille qui est connue pour ses opinions. Fernand est suspecté d'être un résistant. Les soldats allemands, qu'ils hébergent malgré eux, s'opposent un jour, au nom du règlement, à une perquisition opérée par des gendarmes français accompagnés d'inspecteurs en civil. Les gendarmes n'ont pas le droit de fouiller les logis où se trouvent des Allemands. Si la fouille avait eu lieu, ils auraient trouvé un paquet de tracts caché dans l'une des cabanes à lapins. Suite à cet incident, l'un d'eux a proposé à Fernand de dissimuler sous son lit ce qu'il avait à cacher. Se doutaient-ils des contenus? Les trois soldats sur le départ se sont fait tirer le portrait et ont laissé une photo au couple lorsque le pont a été construit fin novembre 1941.

Cette micro-histoire écrite par Georges Waysand a le mérite de documenter très précisément un pan de la recherche resté bien trop en retrait: celui de la résistance juive, tout en montrant à quel point les liens pouvaient être étroits entre la Belgique et le Nord de la France dans le MBB. Et à la question «Etaient-ils des communistes juifs ou des juifs communistes ? » 15 , l'auteur répond ainsi : «Ils n'étaient pas nés communistes, ils l'étaient devenus, mais juifs ils étaient nés, même laïcs, ils l'étaient par leur culture et les regards des autres. L'adhésion était une décision, les conditions initiales un donné intangible » (p. 85). L'itinéraire de Jean-Mojsze et Esther-Estoucha ne peut se dissocier d'un engagement antifasciste précoce, puis communiste en Belgique, auprès des républicains espagnols dans les Brigades internationales pour elle, ce qui les amènera presque inéluctablement - dans la Résistance, lui qui fut fusillé au Fort du Vert-Galant et elle déportée à Ravensbrück et Mauthausen. Loin de souscrire à un « devoir de mémoire » insipide et convenu, il a redonné vie à ces «illégaux», à ces «étrangers

\footnotetext{
${ }^{14}$ L'UJRE est une organisation juive française née en 1943 dans la Résistance et liée à la réorganisation de la section juive de la Main d'Euvre Immigrée (MOI).

${ }^{15}$ Voir les travaux d'Annette Wieviorka : Ils étaient juifs, résistants, communistes, Paris, Perrin, 2018, 480 p. et ceux d'Yvan Jablonka: Histoire des grands-parents que je n'ai pas eus. Une enquête, Paris, Le Seuil, 2012, 448 p.
} 
indésirables ». Il donne un écho tout particulier à notre époque toujours en proie à l'antisémitisme, à la xénophobie.

En replay: France culture, 28 octobre 2020, Émission Esprit de justice, Le père interrompu.

https://www.franceculture.fr/emissions/esprit-de-justice/le-pere-interrompu

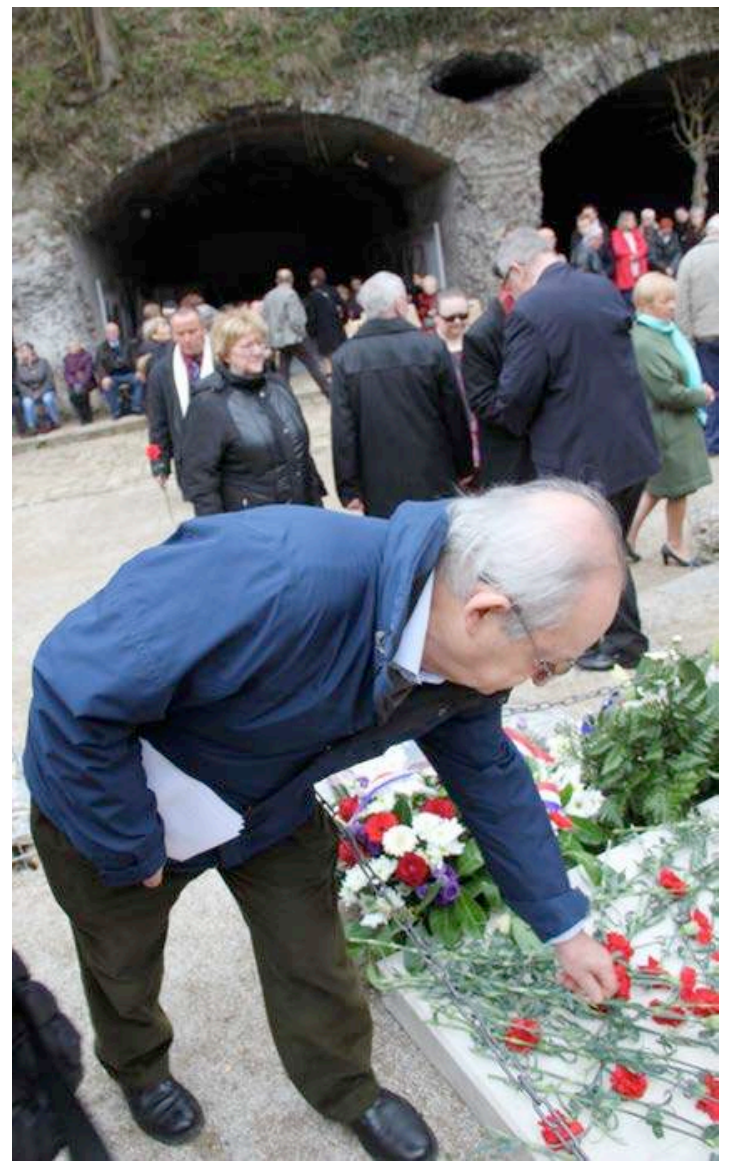

Cérémonie commémorative au fort du Vert-Galant, Wambrechies (Nord), 2015

Georges Waysand dépose un œillet au pied de la stèle commémorative en hommage aux fusillés parmi lesquels se trouve son père

(Photo Monique Heddebaut) 


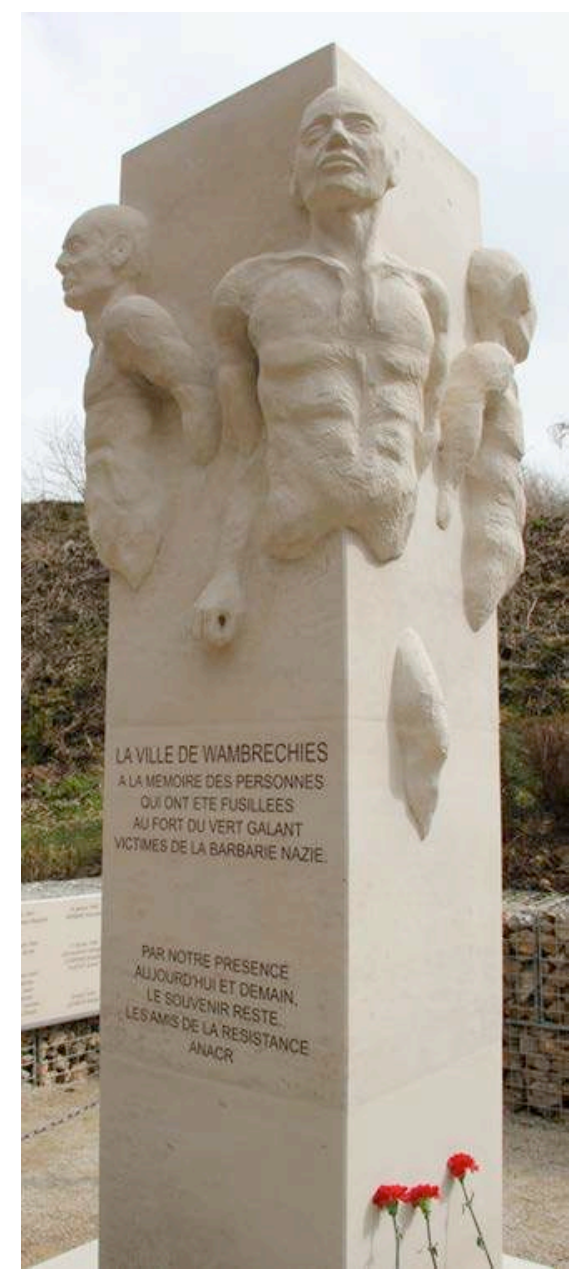

Stèle commémorative érigée en 2012 et créée par l'artiste allemand Ilja Wedekind en hommage aux fusillés du Vert-Galant à Wambrechies (Nord)

(Photo Monique Heddebaut) 